Check for updates

Cite this: RSC Adv., 2017, 7, 19007
Received 20th December 2016 Accepted 14th March 2017

DOI: $10.1039 / c 6 r a 28442 f$

rsc.li/rsc-advances

\section{Predicting human intestinal absorption with modified random forest approach: a comprehensive evaluation of molecular representation, unbalanced data, and applicability domain issues $\uparrow$}

\author{
Ning-Ning Wang, $\dot{+}^{\mathrm{a}}$ Chen Huang, $\dot{+}^{\mathrm{b}}$ Jie Dong, ${ }^{\mathrm{a}}$ Zhi-Jiang Yao, ${ }^{\text {ac }}$ Min-Feng Zhu, ${ }^{\text {ac }}$ \\ Zhen-Ke Deng, ${ }^{a}$ Ben LV, ${ }^{c}$ Ai-Ping Lu, ${ }^{d}$ Alex F. Chen ${ }^{\text {ac }}$ and Dong-Sheng Cao (D) *acd
}

\begin{abstract}
With the increase of complexity and risk in drug discovery processes, human intestinal absorption (HIA) prediction has become more and more important. Up to now, some predictive models have been constructed to estimate HIA of new drug-like compounds with acceptable accuracies, but there are still some issues to be explored including the limited and unbalanced HIA data, the performance of different types of descriptors and the application domain issues of published models. To address these problems, in this study, we collected a relatively large dataset consisting of 970 compounds, and 9 different types of descriptors were calculated for further modeling. For all the modeling processes, a parameter named samplesize in the random forest (RF) method was applied to balance the dataset. And then, classification models were established based on different training sets and different combinations of descriptors. After a series of modeling processes and various comparisons among these statistical results, we explored the aforementioned problems and evaluated the reliabilities of existing HIA classification models and subsequently obtained a robust and applicable model based on a combination of 2D, 3D, $\mathrm{N}^{+}$and $\mathrm{N}_{\text {rule-of-five }}$ (for the training set, $\mathrm{SE}=0.892, \mathrm{SP}=0.846$; for the test set, $\mathrm{SE}=0.877, \mathrm{SP}=0.813$ ). Compared with other published models, our model exhibits some advantages in data size, model accuracy and model practicability to some extent. This structure-activity relationship model is necessary and useful for HIA prediction and it could be a convenient tool for virtual screening in the early stage of drug development.
\end{abstract}

\section{Introduction}

In recent years, complexity and risk have increased greatly in drug discovery and development processes which result in increasing investment in drug research. ${ }^{1}$ For an approved drug, this investment has increased from 800 million dollars in 2003 to 2.6 billion dollars in 2014 . The decline in the productivity of the pharmaceutical industry is mostly due to the poor ADMET (absorption, distribution, metabolism, excretion, and toxicity) properties. ${ }^{2-9}$ Nowadays, oral administration has become the route favored by patients because of its ease and patient

${ }^{a}$ Xiangya School of Pharmaceutical Sciences, Central South University, Changsha, 410013, P. R. China. E-mail: oriental-cds@163.com

${ }^{b}$ School of Mathematics and Statistics, Central South University, Changsha 410083, P. R. China

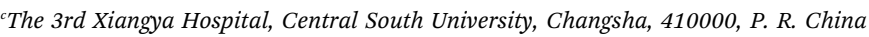
${ }^{d}$ Institute for Advancing Translational Medicine in Bone \& Joint Diseases, School of Chinese Medicine, Hong Kong Baptist University, Hong Kong SAR, P. R. China

$\dagger$ Electronic supplementary information (ESI) available. See DOI: 10.1039/c6ra28442f

$\ddagger$ The first two authors equally contribute to this paper. compliance. For a new oral drug, bioavailability is one of the most desirable attributes, whereas the determination of oral bioavailability is very challenging due to the fact that bioavailability is a complex function of many biologic and physicochemical factors. ${ }^{\mathbf{1 0 1 1}}$ The relationship between oral bioavailability and intestinal absorption has been proven by previous studies and we can draw the conclusion that the bioavailability of most compounds (64\%) is mainly controlled by the intestinal absorption process. ${ }^{12}$ Consequently, the aforementioned phenomenon reminds us that human intestinal absorption (HIA) could be an alternative indicator for oral bioavailability to some extent and thus it also plays an important role in preclinical drug evaluation.

Currently, preclinical HIA screening strategies could be classified into three categories according to different methods. In the early stage of HIA study, the evaluation of HIA was generally based on animal experiments. ${ }^{13,14}$ But considering animal protection and research status, it is necessary to develop some alternative methods to evaluate HIA. Subsequently, diverse quantitative structure-activity relationship (QSAR) models for in vitro permeability were established to estimate 
HIA. Among these methods, the most widely used ones are parallel artificial membrane permeability assay (PAMPA), ${ }^{\mathbf{1 5}}$ human colon adenocarcinoma (Caco-2) cell lines, ${ }^{\mathbf{1 6}}$ and the Madin Darby canine kidney (MDCK) cell. ${ }^{6,17,18}$ Especially, some studies have shown that there is a good sigmoidal relationship between human oral drug absorption and Caco-2 permeability but this relationship seems not so clear. ${ }^{19-22}$ Hou et al. have confirmed that Caco-2 cell lines can be used as a predictive tool to estimate oral absorption just for compounds with good intestinal absorption. While for compounds with low or medium absorption, the Caco- 2 cell lines may not give a very good rank for estimating oral absorption. ${ }^{12,20}$ So even if the Caco-2 permeability predictive models have developed a lot, a high-quality and practical HIA predictive model is still in demand. To achieve this goal, major efforts have been made to build a satisfactory HIA predictive model. First of all, Lipinski proposed a "rule of five" based on several critical properties to identify compounds with possible poor absorption and permeability. ${ }^{23}$ After that, not only regression models, but also classification models have been developed a lot. For regression models, the dataset is always growing and its size ranges from 67 to $619 .^{24,25}$ These models are constructed based on different types of descriptors, such as physicochemical descriptors, ${ }^{\mathbf{1 2 , 2 6 - 2 9}}$ topological descriptors and so on. ${ }^{30-33}$ With regards to computational techniques, the most common mathematical methods include artificial neural networks ${ }^{27,28,33}$ and multiple linear regression. ${ }^{30}$ Furthermore, support vector machine (SVM), ${ }^{\mathbf{2 9 , 3 4}}$ multivariate adaptive regression splines ${ }^{24}$ and stepwise regression $^{25}$ have also been applied. Considering the fact that the distribution between a high-absorbed and poor-absorbed compound is bias, a number of classification models were established in recent years. For these classification models, the number of compounds ranges from 202 to 685 . Other than these descriptors used in regression models, ${ }^{1,13,35,36}$ chargerelated descriptors such as hydrogen bonding capacity and charged partial surface were proposed to improve the predictive ability for HIA models. ${ }^{37}$ In regard to statistical methods, linear discriminant analysis, ${ }^{1,30} \mathrm{SVM}^{35,36}$ and Bayesian ${ }^{37}$ are the most commonly used ones in all the published classification models studies. In 2012, Taravat explained the impact of data distributions for modelling of passive intestinal absorption and attempted to address this problem of unbalanced data distribution by creating a training set through under-sampling the highly absorbed compounds. Their classification model has an accuracy of 0.798 for the training set and an accuracy of 0.958 for the test set. ${ }^{25}$ Based on the same dataset, Hou et al. and Shen et al. also built HIA classification models in 2007 and 2010 respectively $(\mathrm{ACC}=0.97 / 0.98$ for training set; $\mathrm{ACC}=0.98 / 0.99$ for test set). ${ }^{12,36}$ In addition, there have also been several QSAR models with similar statistical results for HIA classification in the last few years. ${ }^{13,37}$

Although researchers have obtained some acceptable HIA predictive models based on size-different datasets, there are still some issues to be explored. First is the bias distribution of available HIA data. For the existing models, the predictive ability for poor-absorbed compounds is usually worse than that for high-absorbed compounds due to the lack of negative molecules. Second are the descriptors used in the modeling process. Various types of descriptors have been applied to construct models as previously mentioned; we wonder which one or ones would perform better for HIA prediction. Third are the application domain and reliability of the present models. We have noticed that existing models have distinctly different predictive accuracies and those models based on Hou's dataset performed much better than models before and after them. Therefore, it is necessary to evaluate HIA classification models based on Hou's dataset through rebuilding models.

To address the above-mentioned issues, we collected a relatively large HIA dataset from the existing published literature and 9 types of descriptors were calculated for them primarily. In addition, a parameter named sample size in random forest (RF) was proposed to balance the dataset in the modeling process. Overall, there are mainly two purposes of this paper: (1) to evaluate previous HIA classification models by rebuilding HIA models and external test set and (2) to obtain a more applicable model with an optimized RF method based on a combination of different descriptors.

\section{Materials and methods}

\section{Data collection}

The dataset in the present study consists of 970 drug and druglike compounds. Among them, there were 856 real drugs and the other 114 "drug-like" compounds were filtered by the "Lipinski rule of five". We collected their HIA values from 13 HIA studies. ${ }^{1,3,13,20,24,26,27,32,37-41}$ To improve the quality and reliability of the dataset, we dealt with it as follows. When there were two or more entries for one molecule, if these values did not differ a lot $(<20 \%)$, the arithmetic mean value was adopted to reduce the random error; otherwise, the corresponding HIA value will be reconfirmed again. Solvent or saline ions adhering to the molecules were removed automatically by Molecular Operating Environment software (MOE, version 2014). At the end, the dataset consists of 955 numerical and 15 categorical fraction absorption values. And then, a HIA\% value of $30 \%$ was used as the criterion to divide chemical agents into the goodabsorption (HIA $(+)$ ) and the poor-absorption (HIA (-)) classes. ${ }^{35}$ For the present dataset, the HIA $(+)$ set and HIA $(-)$ set have 818 and 152 compounds respectively.

To verify the reliability and predictive ability of models, all compounds were divided into a training set (776 compounds) and a test set (194 compounds) using MOE according to their chemical structure. ${ }^{42}$ To further validate the generalization ability of our model, we applied Shen's dataset (634 oral drugs) and Hou's dataset (1013 bioavailability values) as the external validation datasets. ${ }^{36,43}$ After eliminating the duplicates, 267 oral drugs and 175 bioavailability values were obtained. Considering the fact that the value of HIA is always greater than oral bioavailability, the compounds that have bioavailability values exceeding $30 \%$ and those drugs with oral dosage formulations were considered to be included in HIA + external test set. These compounds and corresponding HIA labels can be found in the ESI. $\dagger$ 


\section{Molecular descriptor calculation}

In the present study, 9 types of molecular descriptors were calculated, namely two-dimensional (2D) descriptors, threedimensional (3D) descriptors, molecular fingerprints (ECFP2; ECFP4; ECFP6; FP2) and structural fragments (FP4; Estate; MACCS). Firstly, all the compounds were corrected using the "wash" function in MOE. And then, compounds were optimized to obtain 3D structures by MMFF94x (Merck Molecular Force Field 94X, eps $=r$, cutoff $[8,10]$ ) and the gradient-threshold of potential energy was set to $0.001 \mathrm{kcal}^{-1} \mathrm{~mol}^{-1} .^{44}$ After that, 192 2D descriptors and 117 3D descriptors were computed by MOE. Moreover, ChemDes was applied to calculate these fingerprints and structural fragments. ${ }^{45}$ Thus, 1024 ECFP2/ECFP4/ECFP6/ FP2 fingerprints; 307 FP4 fragments; 79 Estate fragments; and 167 MACCS fragments were prepared. Besides the abovementioned descriptors, we recommended another two descriptors that seem to make sense in previous studies. ${ }^{35,36}$ One is the number of violations of the four rule-of-five rules ( $\left.N_{\text {rule-of-five }}\right)$ developed by Lipinski, ${ }^{46}$ which was calculated by ChemoPy. ${ }^{47,48}$ The other is $\mathrm{N}^{+}$, which was used to represent the existence of a positively charged $\mathrm{N}$ atom. If $\mathrm{N}^{+}$was found in the molecule, the label was defined to be 1 ; otherwise, the label was defined to be 0 .

\section{Modified random forest and feature selection}

Recent studies have suggested that RF offers several striking features which make it very attractive for QSAR/QSPR studies. ${ }^{\mathbf{9}}$ These include relatively high accuracy of prediction, built-in descriptor selection, and a method for assessing the importance of each descriptor to the model. RF was applied to build HIA classification models in this study and it was used in the statistical computing environment $\mathrm{R}^{{ }^{50-53}}$ Optimization of the training parameters was performed using $R$ scripts which iteratively changed each parameter one-by-one and regenerated the classification model. The ranges for three main parameters to select the optimum values were as follows: ntree was set to 500; nodesize was set to 1 ; and mtry was in the range $(2,12)$ for the $2 \mathrm{D}$ and $3 \mathrm{D}$ descriptors and for the fingerprint descriptors it was in the range of $(6,58)$.

Due to the unbalanced dataset, the obtained models may be biased if general modeling processes were applied. ${ }^{54,55}$ To obtain some more balanced classification models, we applied a parameter named sample size to achieve this goal. Sample size was used to determine the number of HIA $(+)$ compounds and HIA (-) compounds in the process of modeling. For example, when the sample size is set to $(100,200)$, it means that 100 poorabsorbed compounds and 200 high-absorbed compounds were randomly selected to build a tree in each modeling process and this process repeated many times to guarantee that every compound in the training set could be used in the final RF model. The use of sample size guarantees that the number of positive samples and negative samples is relatively balanced in each bootstrap sampling process.

For $2 \mathrm{D}$ and $3 \mathrm{D}$ descriptors, two pretreatments were performed to delete some uninformative descriptors before further feature selection: (1) delete the descriptors whose variance is
0 or approaches 0 ; (2) if the correlation coefficient between two descriptors is higher than 0.95 , only one was reserved. After the preliminary descriptor pruning, further feature selection was as follows. Firstly, all descriptors were applied to build a classification model and these involved descriptors were sorted according to their importance. Then, the last two descriptors were removed and the rest were used to rebuild the model and a new descriptor order was obtained. This process was repeated until the last two remaining descriptors were used for modeling, and finally we get a series of models based on different numbers of descriptors. Among them, we can choose a best feature combination according to the number of descriptors and the error value of the model.

\section{Performance evaluation}

To ensure that the derived model has good generalization ability, five-fold cross validation and an individual test set were used for the validation purpose. ${ }^{6}$ The performances of the HIA classification models were evaluated using the following statistical parameters: true positive (TP); false negative (FN); true negative (TN); false positive (FP); sensitivity (SE); specificity $(\mathrm{SP})$; accuracy (ACC); $F$ value $(F)$; area under receiver operating characteristic curve (AUC); Matthews correlation coefficient (MCC). Additionally, to deal with the possible bias from unbalanced data, we proposed $\mathrm{SP} \times \mathrm{SE}$ to optimize the $\mathrm{RF}$ model parameters and measure the whole predictive performance of the HIA classification models. These classification evaluation parameters are defined as follows:

$$
\begin{gathered}
\mathrm{SE}=\frac{\mathrm{TP}}{\mathrm{TP}+\mathrm{FN}} \\
\mathrm{SP}=\frac{\mathrm{TN}}{\mathrm{TN}+\mathrm{FP}} \\
\mathrm{ACC}=\frac{\mathrm{TP}+\mathrm{TN}}{\mathrm{TP}+\mathrm{FP}+\mathrm{TN}+\mathrm{FN}} \\
\mathrm{FCC}=\frac{\frac{2 \mathrm{TP}}{2 \mathrm{TP}+\mathrm{FP}+\mathrm{FN}}}{\sqrt{(\mathrm{TP}+\mathrm{FN})(\mathrm{TP}+\mathrm{FN})(\mathrm{TN}+\mathrm{FN})(\mathrm{TN}+\mathrm{FP})}}
\end{gathered}
$$

\section{Results and discussion}

\section{Evaluation of models based on Hou's dataset}

1.1 Preliminary evaluation of models based on Hou's dataset. Based on this HIA dataset $(480+98)$, a series of HIA classification models were developed in this part. Sample size was set to $(60,80)$ in this modeling process and the statistical results of cross validation and test set validation are shown in 


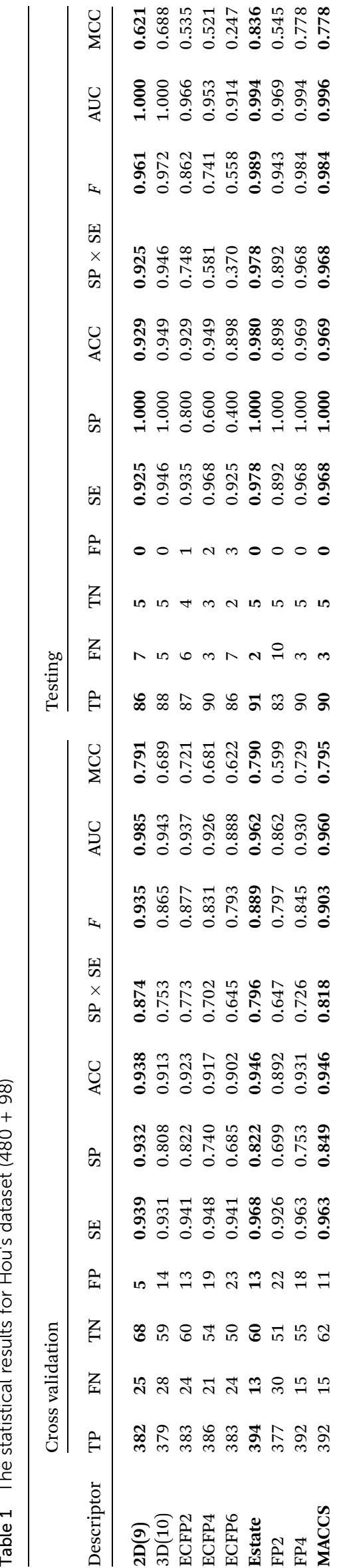

Table 1. From the table, we can find that the best three models were derived from 2D descriptors, Estate and MACCS structural fragments (highlighted in bold). These three predictive models perform as well as those reported in the literature (Tr: 0.98/0.95; Te: $0.98 / 1$ ). To ensure the reasonable performance of HIA classification model built by RF, 1000 times of training-validation procedure were conducted. For every classification model, one-fifth of the positive compounds and one-fifth of negative compounds were randomly selected as the validation set and the rest composed the training set. Sample size was set to (60, 80) uniformly in the modeling processes. The distribution diagrams of accuracy, sensitivity and specificity for the validation set of 1000 randomized models can be seen in Fig. 1. From the figure, we can clearly see that sensitivity, specificity, and accuracy of these randomized models were mainly in the range of $0.954 \pm 0.02,0.900 \pm 0.07$, and $0.947 \pm 0.02$, respectively. Compared with the statistical results of these random models, the models based on Hou's dataset and different descriptors have reasonable performance. To put it another way, this result also indicated that our descriptors and the proposed modeling methods for HIA classification dataset were satisfactory.

There was a strange fact that the predictive ability for the poor-absorbed compounds was better than that for goodabsorbed compounds in the test set. This was not consistent with our common sense. Several studies have reported the difficulty of predicting poor-absorbed compounds due to the unbalanced issue of HIA datasets. ${ }^{13,25,37}$ In these studies, the specificity values of their classification models were near 0.600.70 and always smaller than their sensitivity values. After examining the original dataset, we found that there were 73 poor-absorbed compounds in the 480 training molecules but only 5 poor-absorbed compounds in the 98 test molecules.
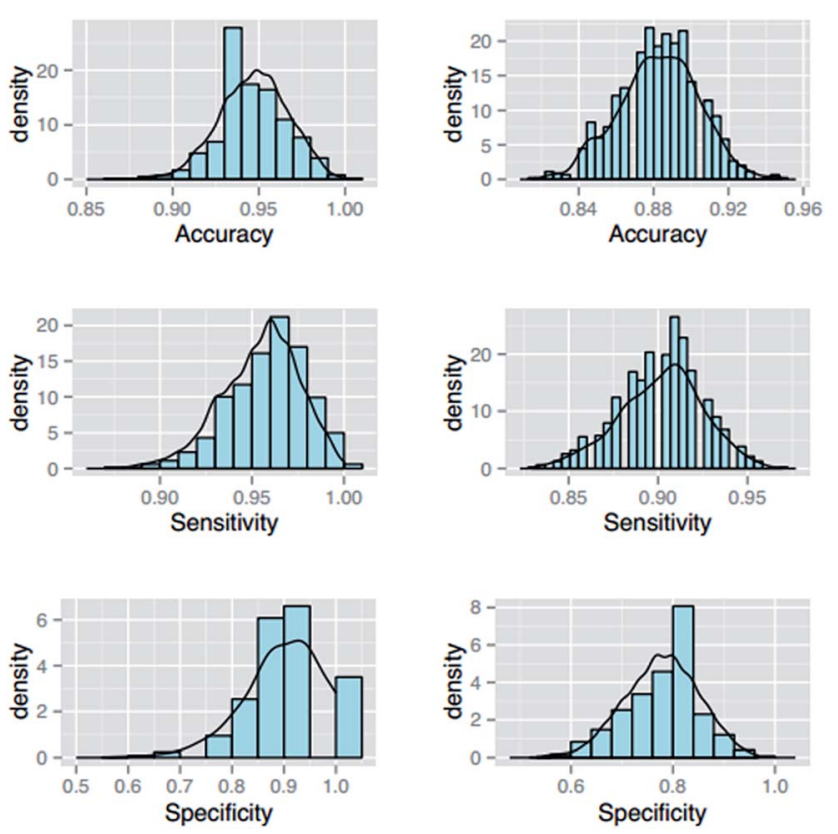

Fig. 1 Distribution diagrams of accuracy, sensitivity and specificity for 1000 test sets (the left-hand three are based on 578 compounds; the right-hand three are based on 970 compounds). 
Hence, we considered that the number of poor-absorbed compounds in the test set was too little to reflect the actual predictive ability of the HIA classification model, especially for the specificity.

1.2 Further validation for models based on Hou's dataset. To further validate model performance based on Hou's dataset, we split our collected data into a training set of 578 compounds and a test set of 392 compounds. In this part, sample size was set to $(60,70)$. The corresponding results are displayed in Table 2. From the table, the best three were also models built with 2D descriptors, Estate and MACCS structural fragments on the whole (highlighted in bold). As a whole, the predictive ability for the cross validation set was similar to those models based on 480 compounds, but predictive ability for the test set was much worse, especially for the poor-absorbed compounds. For the test set, the sensitivity ranged from 0.806 to 0.873 but the specificity ranged from 0.552 to 0.628 which was much smaller than the sensitivity value. From the aforementioned results, we can draw the conclusion that the models based on Hou's dataset have an acceptable predictive ability for the external good-absorbed compounds, but it seems not so practical for the external poor-absorbed compounds. Taking dataset, descriptors and statistical methods into consideration, we speculated that the structural diversity and the unbalance of the HIA dataset may account for a large part of this unfavorable result.

\section{HIA classification models built with new training set (776 compounds)}

2.1 Modeling process for individual type of descriptor. To avoid the aforementioned possible issues and obtain a practical classification model, we constructed HIA classification models based on 776 compounds and their statistical results are displayed in the Table 3. From the table, we can draw the conclusion that the best three classification models were built with 2D descriptors, Estate and MACCS fragments on the whole (highlighted in bold). For the training sets in the three best models, the sensitivity ranged from 0.883 to 0.906 and the specificity value ranged from 0.761 to 0.821 . For the test sets, the sensitivity ranged from 0.846 to 0.883 and the specificity value was in the range of 0.719-0.781. Compared with the models in the first mentioned case, the cross validation results were a little worse. Nevertheless, the statistical results of the external validation in the test set were much better, especially the specificity. It demonstrated that the obtained models were more practical in predicting the HIA classification for new compounds than those derived from Hou's dataset. Moreover, it may also be strong evidence for the poorer structural diversity of Hou's dataset as described above.

To further ensure the reasonability of our model, 1000 randomized predictive models have also been obtained based on 970 compounds and the distribution diagrams of accuracy, sensitivity and specificity for the validation set are also shown in Fig. 1. In this process, sample size was set to $(90,100)$ uniformly. From the figure, we can see that the accuracy values of these randomly shuffled models were mainly located in the range of $0.883 \pm 0.02$. Subsequently, their sensitivity values were mainly 


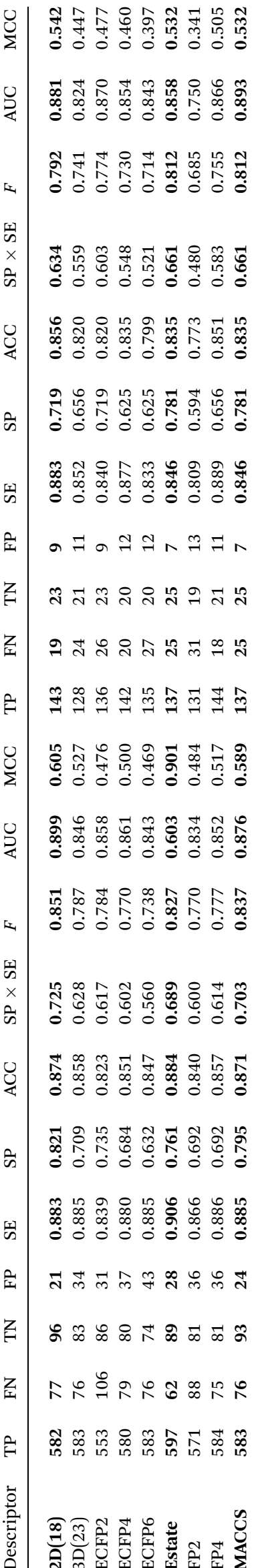

in the range of $0.902 \pm 0.02$ and the specificity values were gathered in the range of $0.778 \pm 0.072$. Compared with the statistical results from our predictive model and those derived from Hou's dataset, it is apparent that our primary models were more rational than other models from the perspective of subdivision for the training set and test set. These results also indicated the importance of the creation of training set and test set for a modeling process.

2.2 Modeling process for different combined descriptors. In this part, we aimed at obtaining a more robust HIA classification model through a combination of different types of descriptors. From the statistical results from individual descriptors, we can see that both of the models based on 2D and $3 \mathrm{D}$ descriptors have better results than other models from an overall perspective. But for those models based on fingerprint and structural fragments, only the two from Estate and MACCS performed as well as the $2 \mathrm{D}$ - and $3 \mathrm{D}$-based classification models. Therefore, we decided to combine the following four types of descriptors for further study: 2D descriptors; 3D descriptors; Estate; and MACCS fragments. In addition, $\mathrm{N}^{+}$and $N_{\text {rule-of-five }}$ were also added to the model. All in all, there are 9 predictive models based on different descriptor combinations and their statistical results are displayed in Table 4.

From the table, some interesting phenomena were drawn out. Firstly, compared with the model based on only $2 \mathrm{D}$ descriptors, the model derived from a combination of 2D and $3 \mathrm{D}$ descriptors has better performance for both training and test set, especially for specificity ( $\mathrm{SP}=0.838 / 0.750$ ). This may imply that the $3 \mathrm{D}$ descriptors could give some ESI $\dagger$ for HIA prediction. Secondly, after adding $\mathrm{N}^{+}$, the predictive performance for the test set improved to some extent (SP: 0.750 to 0.813). We speculated that this is in accordance with previous studies which have demonstrated that a compound with $\mathrm{N}^{+}$has poor intestinal absorption generally. Similarly, the new model with $N_{\text {rule-of-five }}$ also has a better predictive ability. Thirdly, for the other classification models based on combinations of $2 \mathrm{D}$ / 3D and structural fragments, the specificity values were smaller for both training set and test set after adding structural fragments. This result was probably because the predictive model from the individual type of structural fragment generally has a relatively poor specificity value. Fourthly, the statistical results for models based on $2 / 3 \mathrm{D}+\mathrm{E}$ and $2 / 3 \mathrm{D}+\mathrm{E}+\mathrm{M}+\mathrm{N}+\mathrm{R}$ were the same, which may imply that the Estate fragments cover the useful information contained in MACCS fragments, $\mathrm{N}^{+}$and $N_{\text {rule-of-five }}$ In short, the final HIA classification model (highlighted in bold) derived from seven 2D descriptors, two 3D descriptors, $\mathrm{N}^{+}$and $N_{\text {rule-of-five }}$ was the best one after a series of attempts for descriptor combination. In addition, we also rebuilt models based on the same descriptors with $2 ; 3+$ NR and 2; 3 + EMNR models without an optimized sample size in RF; their results are also shown in Table 4. The two models have excellent abilities to predict positive compounds but they are very short of predictive abilities for poor-absorbed compounds. Compared with these models using sample size, the importance of a balanced classification model was self-evident.

To define the structural domain the model does not cover, we rebuilt classification models based on the total of 970 


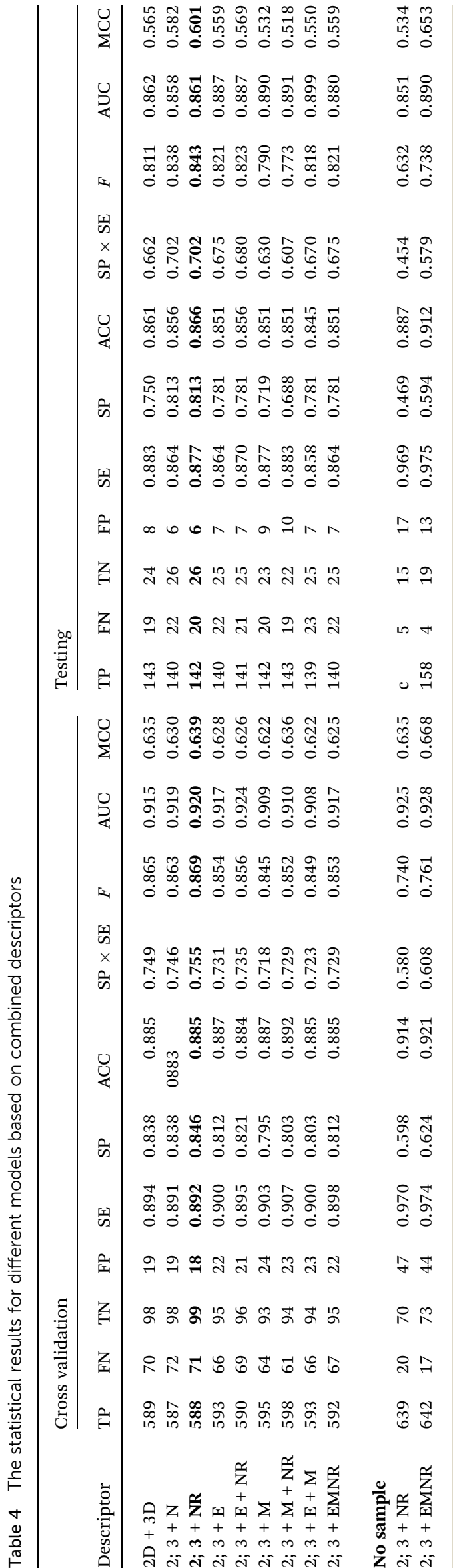

compounds and five-fold cross validation was used to evaluate the quality of a model. This process was repeated 1000 times. From these predictive results, we found some anomalous compounds that always are wrongly categorized by all predictive models. Among them, there were eight representative molecules and their structures are shown in Fig. 2. For the eight compounds, cyclosporine has the largest molecular weight of 1202 and is composed of 11 amino acids. Cyclosporine is a kind of immune inhibitor widely used to prevent organ transplant rejection. ${ }^{56}$ We speculated that the incorrect prediction may due to its particularly large molecular weight and its macrocycle structure. Both azithromycin and viomycin are macrolide antibiotics and they can inhibit protein synthesis through blocking peptide and displacement of mRNA. ${ }^{57}$ This class of antibiotics has a big lactonic ring commonly and it may make HIA prediction much harder. Fosinopril, a new angiotensin converting enzyme inhibitor, is widely used to relieve light, medium and severe hypertension and various types of heart failure. ${ }^{58}$ From its structure, we found that there are an L-proline and a phosphoryl which are unusual in the whole dataset. As to the other four compounds, although there is nothing abnormal from their 2D structures, their partial charges and potential energies or other properties may not be in the application domain of the predictive model. After removing these compounds from the initial dataset, we obtained a new classification model: $\mathrm{TP}=583, \mathrm{FN}=76, \mathrm{TN}=100, \mathrm{FP}=17, \mathrm{SP}=$ $0.855, \mathrm{SE}=0.885, \mathrm{ACC}=0.880, \mathrm{AUC}=0.921$.

2.3 Scaffold analysis of the proposed datasets. To evaluate the diversity of molecular structures, we performed a scaffold analysis for the two datasets. The scaffolds were extracted from compounds by removing all R-groups but retaining linkers between ring systems. And then, carbon skeletons were derived from scaffolds by changing each heteroatom to a carbon atom and all bond orders to single bonds. Thus, different carbon skeletons represent topologically distinct scaffolds. ${ }^{59,60}$ The detailed information of these scaffolds and carbon skeletons can be found in the ESI. $\dagger$ After the scaffold analysis, we obtained 372 scaffolds and 258 carbon skeletons for Hou's dataset, 576 scaffolds and 392 carbon skeletons for our dataset. According to the analysis result, our dataset has covered almost all chemical structures commonly appearing in drug compounds, from simple straight-chain compounds to macrocyclic compounds, from simple cyclopentane or benzene ring to complex heterocyclic compounds or polycyclic compounds. As a comparison, our proposed dataset showed some advantages in both scaffold number and carbon skeleton number. Specifically, we also analyzed the scaffolds for poor-absorbed compounds in the two datasets. There were 56 scaffolds and 50 carbon skeletons for Hou's dataset, 114 scaffolds and 95 carbon skeletons for our dataset. From the scaffold analysis, we can draw the conclusion that our proposed HIA dataset covers a larger chemical space than the other dataset and the same phenomenon can be found for poor-absorbed compounds specifically. Thus, we speculated that the larger chemical space of the proposed dataset may partly contribute to the better predictive ability of our prediction models. 


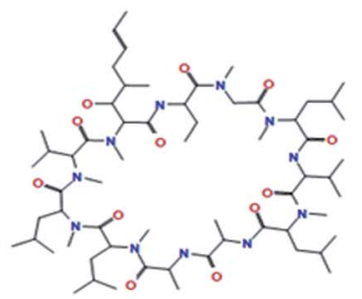

Ciclosporin

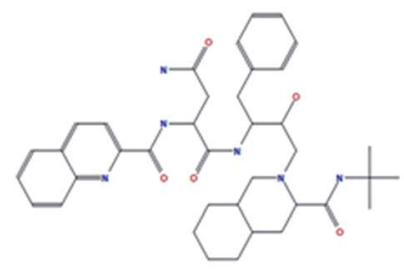

Saquinavir mesylate

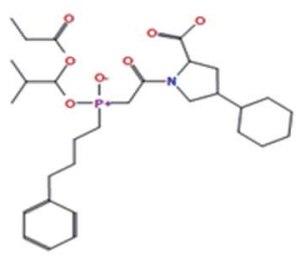

Fosinopril sodium

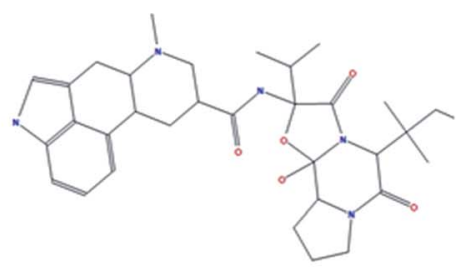

Ergoloid_mesylate

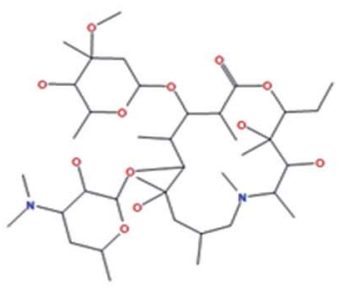

Azithromycin

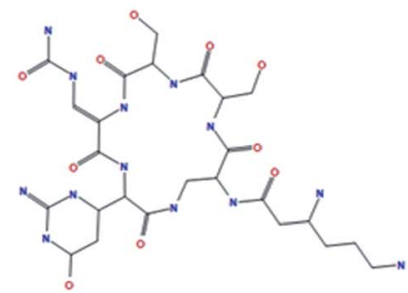

Viomycin

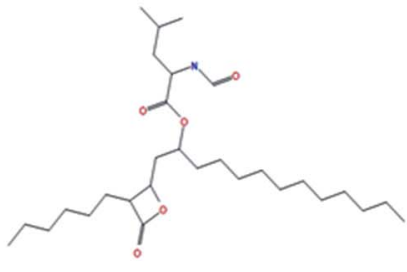

Orlistat

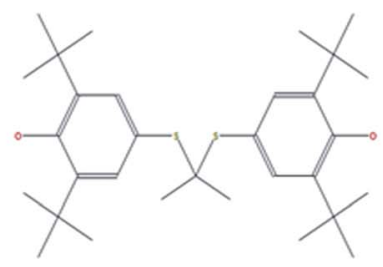

Probucol

Fig. 2 Structures of 8 compounds that always are wrongly categorized.

2.4 External validation for the best HIA classification model. We continue to validate our model by two external validation sets. Table 5 lists the statistical results from two external validation sets. For these 267 oral drugs, 222 compounds were classified correctly and the sensitivity value was 0.831 . For the bioavailability dataset, the model could identify $83.4 \%$ of compounds to the correct category. Although the sensitivity values of the two external datasets were a little smaller than those for the training set and test set, we still deemed the predictive ability of the HIA classification model as relatively satisfactory due to the fact that the decrement was within acceptable limits. Therefore, the classification model proposed by us can be seen as a practical tool in predicting HIA category for new chemical entities.

2.5 Model interpretation. Table 6 lists 11 descriptors used in our final model. Additionally, to clearly visualize the role of each descriptor in classification, the tree visualization from CART is shown in Fig. 3. Clearly, there are several significant descriptors contributing to the prediction of HIA. (1) The numbers of hydrogen bond donors and acceptors and basic atoms play an important role in determining HIA. There are common parameters to represent the hydrogen bond capacity of a compound. When their value for a compound increases, its lipophilicity will be weaker which is detrimental for the compound crossing the cell membrane by passive diffusion. Subsequently, the HIA value of this compound will be decreased.,38,61 (2) KierFlex, a descriptor for molecular flexibility index, is another important element. In essence, it demonstrates the shape and connectivity profiles of a compound and may be

Table 5 The statistical results for the external validation

\begin{tabular}{lllll}
\hline Data & TP & FN & SE & ACC \\
\hline Oral drug & 222 & 45 & 0.831 & 0.831 \\
$F$ & 146 & 29 & 0.834 & 0.834
\end{tabular}

related to structural features such as shape, size, branching and unsaturation. As for these factors, existing literature has emphasized their significant role in HIA prediction. ${ }^{28}$ (3) The five descriptors PEOE_VSA_PPOS, PEOE_VSA_POL, PEOE_VSA_FHYD, PEOE_VSA_FPPOS and DCASA are all dependent on the partial charge of each atom of a chemical structure. We assumed that these descriptors may be related to the hydrogen-bonding capacity and electrostatic contribution on the molecular surface, and consequently can account for the electrostatic interaction between drug molecules and intestine. ${ }^{35}$ Owing to the fact that the CART model has a good ability in predicting highly absorbed molecules $(\mathrm{SE}=0.971)$ but a relatively poor ability for poorly absorbed compounds ( $S P=0.602)$, the descriptor lying at the top of the tree (PEOE_VSA_PPOS) may mainly capture information contained in the high-absorbed compounds. In addition, we can get another finding that

\begin{tabular}{lll} 
Table 6 & Important descriptors involved in final classification model \\
\hline Index & Code & Description \\
\hline 1 & a_donacc & $\begin{array}{l}\text { Number of hydrogen bond donor and } \\
\text { acceptor atoms }\end{array}$ \\
& a_base & $\begin{array}{l}\text { Number of basic atoms } \\
\text { Molecular flexibility index } \\
\text { Total positive polar van der }\end{array}$ \\
3 & KierFlex & $\begin{array}{l}\text { Waals surface area } \\
\text { Total polar van der Waals surface area }\end{array}$ \\
5 & PEOE_VSA_PPOS & $\begin{array}{l}\text { Fractional hydrophobic van der } \\
\text { Waals surface area }\end{array}$ \\
6 & PEOE_VSA_POL & PEOE_VSA_FHYD \\
7 & PEOE_VSA_FPPOS & $\begin{array}{l}\text { Fractional positive polar van der } \\
\text { Waals surface area } \\
\text { Absolute value of the difference between }\end{array}$ \\
8 & DCASA & $\begin{array}{l}\text { CASA+ and CASA- } \\
\text { Solvation energy } \\
\text { Existence of positively charged N atom }\end{array}$ \\
9 & E_Sol & Number of violations of rule-of-five rules \\
10 & $N^{+}$ & $N_{\text {rule-of-five }}$
\end{tabular}




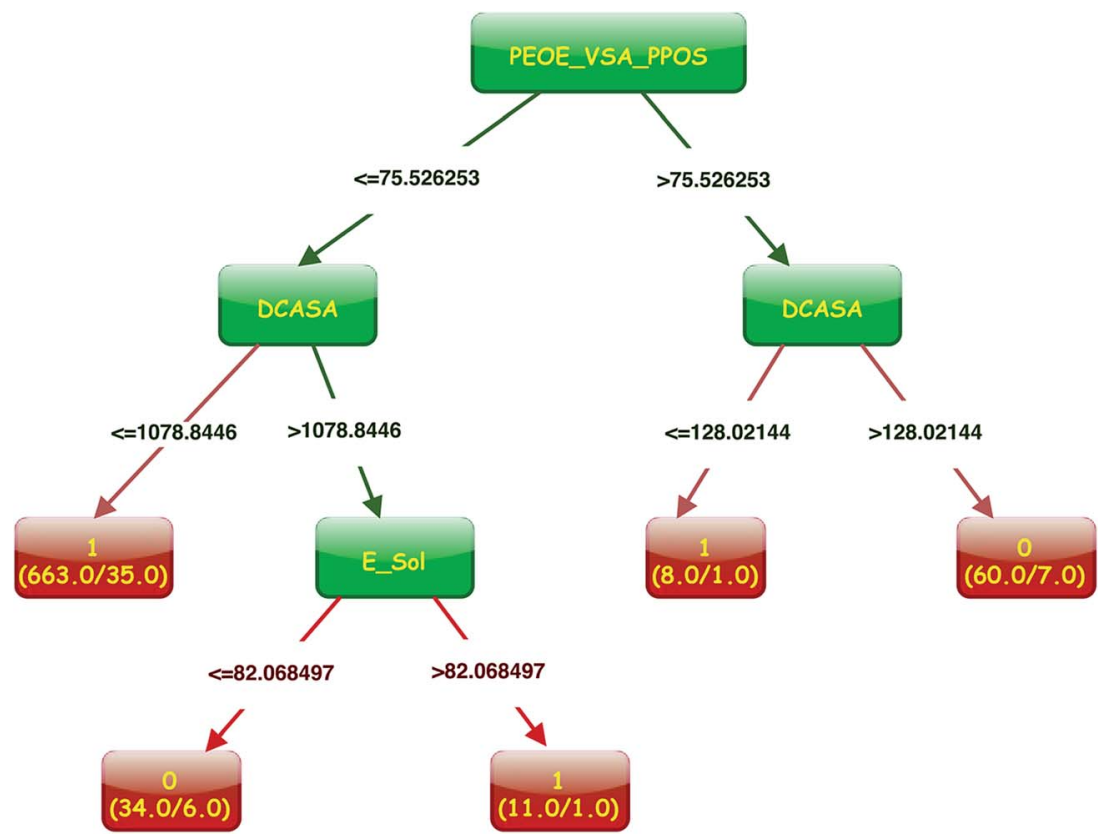

Fig. 3 HIA classification model based on same training set using CART

DCASA has a negative effect on HIA. (4) E_Sol, a potential energy descriptor, was proposed to describe the solvation energy. As is well known, the solvation energy is a decisive factor for a drug molecule in solute process and the interaction process with intestinal epithelial cells. Therefore, it certainly also plays an important role in the prediction of HIA. (5) $\mathrm{N}^{+}$and $N_{\text {rule-of-five }}$ are crucial from a perspective of drug design. It is common sense that molecules with positively charged nitrogen atoms always have very poor intestinal absorptions due to that fact that they are almost dissociative in the gastrointestinal environment. With respect to the $N_{\text {rule-of-five, }}$ it was proposed by Lipinski in 1997 to identify compounds with possible poor absorption and permeability. So after adding this descriptor, the predictive model improved to some extent. In conclusion, except the existence of $\mathrm{N}^{+}$and $N_{\text {rule-of-five, }}$ the hydrogen-bonding capacity, partial charge, solvation energy and the shape of a compound also have great influence on its HIA.

Table 7 lists the first five MACCS and Estate fragments and their corresponding description information. From the table, the first three fragments of MACCS and Estate, which represent the existence of ammonium and oxygen, are the same, indicating that these structural fragments are of high importance for HIA classification. For MACCS 31 and Estate 33, they represent the existence of a positively charged $\mathrm{N}$ atom, only 19/ 119 poor-absorbed compounds have this structural fragment in the training set, all high-absorbed compounds do not have this fragment. For the test set, only 4 compounds have this fragment. Accompanying the above-mentioned descriptors, we speculated that these fragments play a very important role in predicting poor-absorbed drug compounds. In addition to the two fragments, the distributions of the rest were biased to highly absorbed compounds. This is in accordance with the fact that the models derived from MACCS and Estate structural fragments have slightly worse predictive ability for highly absorbed compounds than those derived from other descriptors or fingerprints.

2.6 Comparison with previous HIA classification model. To further evaluate the predictive ability of our balanced HIA model, we collected the HIA classification models from 2000 up to the present. 9 HIA classification models and their performances are displayed in Table 8. Among these predictive models, models 2, 3 and 6 were derived from the Hou's dataset, so their perfect performances in training set and test set may not be so convincing due to the reasons described in Part 1 of this section. There is only one model based on a balanced training set in the remaining 6 HIA models. This model was constructed by Taravat et al. in 2012 with a training set consisting of 94 compounds and its overall accuracies for training set, test set and external validation were $0.872,0.876$ and 0.798 respectively. As a comparison, our HIA model was built based on 776 structurally diverse compounds and its accuracies for training set, test set and external validation were $0.885,0.866$ and $0.831(0.834)$ respectively. Therefore, no matter the dataset size or the predictive performance, our proposed model seems to be better. As to models $1,4,5$ and 7, their predictive abilities for the poor-absorbed compounds were all limited because of the unbalanced dataset (models 1 and 7) or the insufficient number of drug compounds (models 4 and 5). The latest HIA classification model was built by Nikita Basant in 2016 with 403 molecules. A nearly perfect ( $99 \%$ ) classification of the chemicals into two categories by gradient boosted tree was obtained, but it may not be so practical due to the fact that the chemicals in the two classes differ significantly in their characteristics (descriptors) considered for modeling. Above all, our HIA classification model built by optimized RF was satisfactory from the perspectives of data diversity and predictive ability. ${ }^{30}$ 
Table 7 The structural information for the MACCS and Estate descriptors

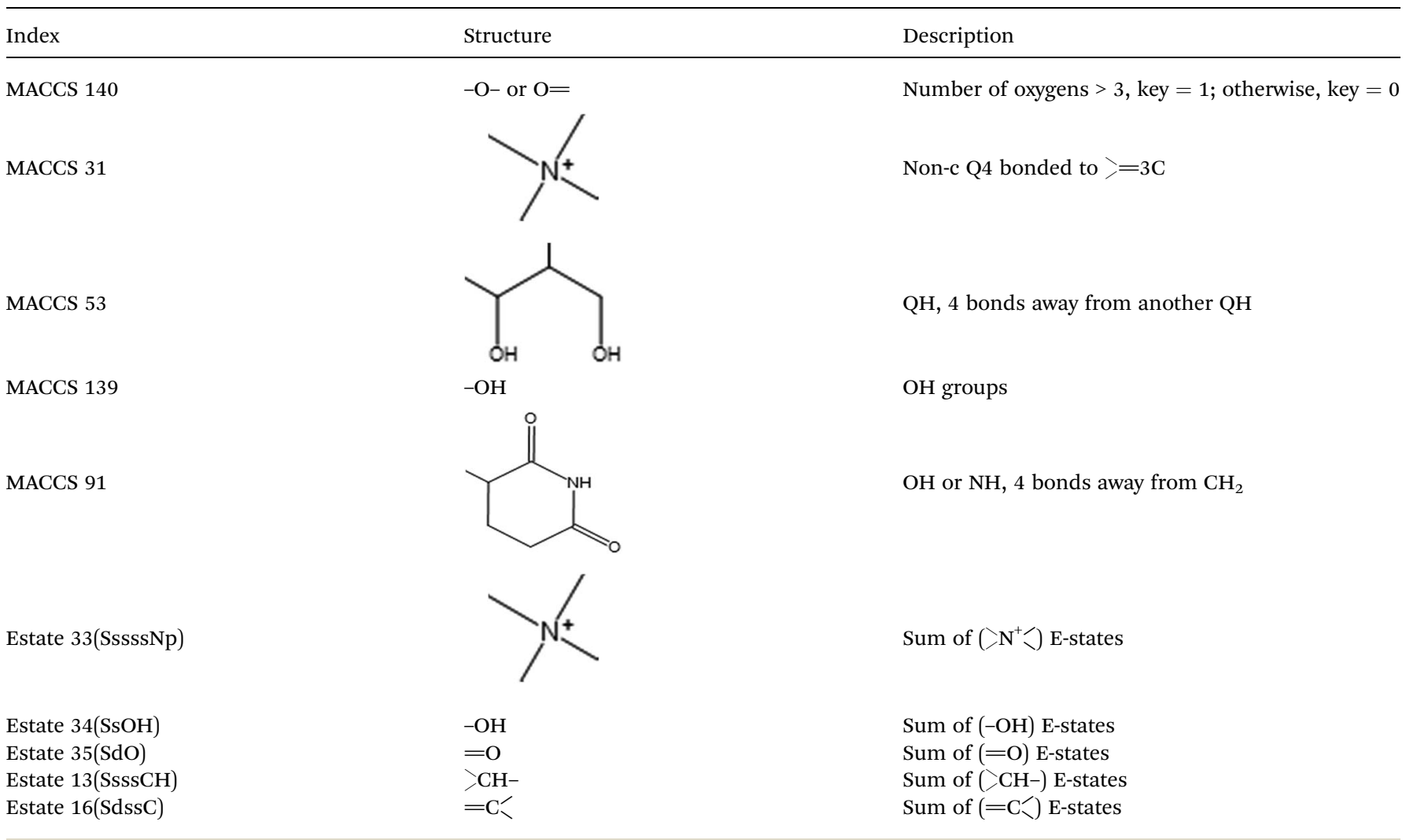

Table 8 Previous HIA classification models and their performances

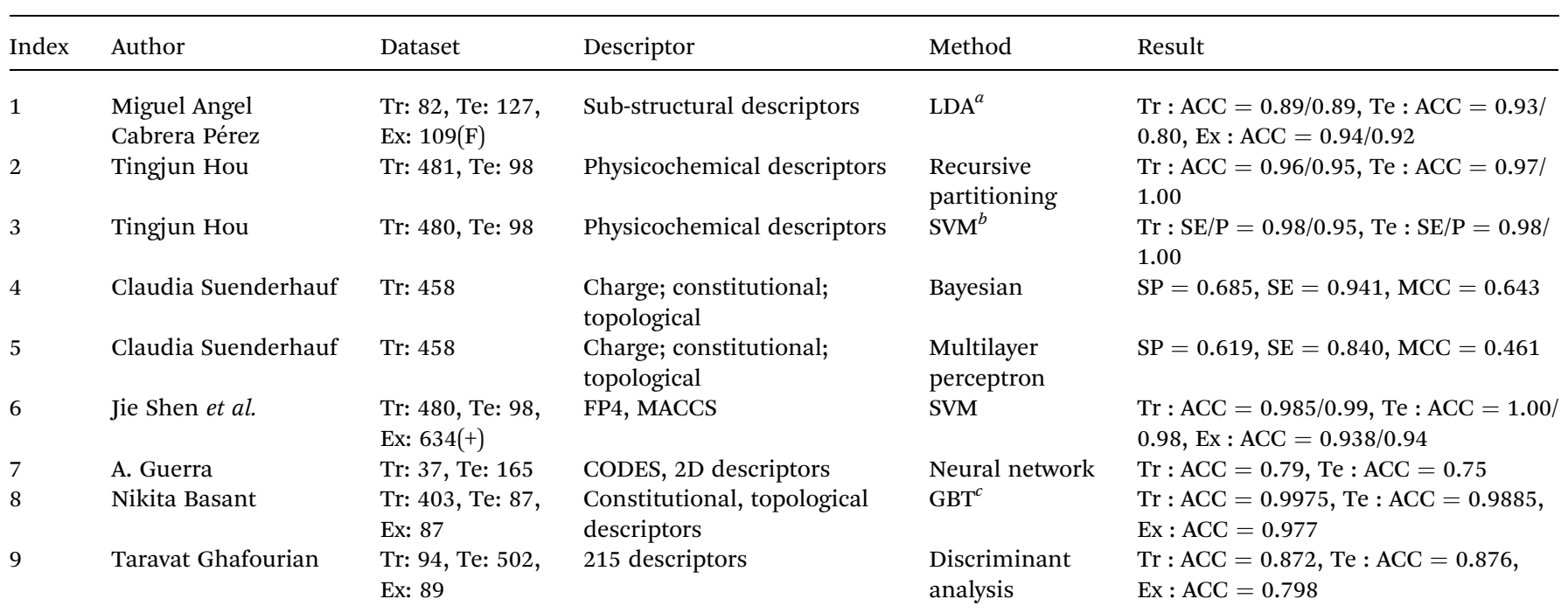

${ }^{a}$ LDA: linear discriminant analysis. ${ }^{b}$ SVM: support vector machine. ${ }^{c}$ GBT: gradient boosted tree.

\section{Conclusion}

In the present study, we collected a relatively large HIA dataset consisting of 970 compounds to evaluate the reliability of HIA classification models based on Hou's dataset and then to obtain a practical classification model. In this process, 9 different types of descriptors and the modified RF model with a parameter named sample size were applied. The obtained results indicated that some previous studies may lead to the over-satisfactory performance for HIA prediction, especially for the poorly absorbed compounds. From these models using 9 types of descriptors, we found that the predictive models based on 2D, 3D, Estate and MACCS descriptors perform better than the others. In addition, sample size is a useful parameter for getting 
a balanced model based on a biased dataset. Finally, we obtained a classification model derived from seven 2D descriptors, two $3 \mathrm{D}$ descriptors, $\mathrm{N}^{+}$and $N_{\text {rule-of-five }}$. Furthermore, the test set and external dataset validated the robustness and reliability of our proposed model. Compared with other published models, our model has some advantages in dataset size, model accuracy and model practicability to some extent. All in all, this study has evaluated the existing HIA models and generated a robust and practical model for HIA classification prediction. It could be a convenient tool for virtual screening in the early stage of drug development.

\section{Declarations}

\section{Availability of data and materials}

All the compounds and their basic structural information and their HIA classification are listed in the ESI. $\dagger$ It includes three training sets and three test sets: $480+98 ; 578+392 ; 776+194$. In addition, two external validation datasets and the detailed information of scaffolds and carbon skeletons can also be found in $\mathrm{ESI}^{\dagger}$ as the external validation datasets.

\section{Conflict of interest}

The authors declare that they have no competing interests.

\section{Acknowledgements}

This work is financially supported by the National Key Basic Research Program (2015CB910700), the National Natural Science Foundation of China (grant no. 81402853), the Central South University Innovation Foundation for Postgraduate (2016zzts498), the Hunan Provincial Innovation Foundation for Postgraduate (CX2016B058), the Project of Innovation-driven Plan in Central South University, and the Postdoctoral Science Foundation of Central South University, the Chinese Postdoctoral Science Foundation (2014T70794, 2014M562142). The studies meet with the approval of the university's review board.

\section{References}

1 M. A. C. Pérez, M. B. Sanz, L. R. Torres, R. G. Ávalos, M. P. González and H. G. Díaz, Eur. J. Med. Chem., 2004, 39, 905-916.

2 B. Booth and R. Zemmel, Nat. Rev. Drug Discovery, 2004, 3, 451-456.

3 S. B. Gunturi and R. Narayanan, QSAR Comb. Sci., 2007, 26, 653-668.

4 T. Hou, J. Wang, W. Zhang, W. Wang and X. Xu, Curr. Med. Chem., 2006, 13, 2653-2667.

5 J.-B. Wang, D.-S. Cao, M.-F. Zhu, Y.-H. Yun, N. Xiao and Y.-Z. Liang, J. Chemom., 2015, 29, 389-398.

6 N.-N. Wang, J. Dong, Y.-H. Deng, M.-F. Zhu, M. Wen, Z.-J. Yao, A.-P. Lu, J.-B. Wang and D.-S. Cao, J. Chem. Inf. Model., 2016, 56, 763-773.
7 J. Dong, Z.-J. Yao, M. Wen, M.-F. Zhu, N.-N. Wang, H.-Y. Miao, A.-P. Lu, W.-B. Zeng and D.-S. Cao, J. Cheminf., 2016, 8, 34.

8 Z.-J. Yao, J. Dong, Y.-J. Che, M.-F. Zhu, M. Wen, N.-N. Wang, S. Wang, A.-P. Lu and D.-S. Cao, J. Comput.-Aided Mol. Des., 2016, 30, 413-424.

9 D.-S. Cao, J. Dong, N.-N. Wang, M. Wen, B.-C. Deng, W.-B. Zeng, Q.-S. Xu, Y.-Z. Liang, A.-P. Lu and A. F. Chen, Chemom. Intell. Lab. Syst., 2015, 146, 494-502.

10 T. Hou and X. Xu, Curr. Pharm. Des., 2004, 10, 1011-1033.

11 T. Kennedy, Drug discovery today, 1997, 2, 436-444.

12 T. Hou, J. Wang, W. Zhang and X. Xu, J. Chem. Inf. Model., 2007, 47, 208-218.

13 A. Guerra, N. E. Campillo and J. Páez, Eur. J. Med. Chem, 2010, 45, 930-940.

14 C. Clemedson, A. Kolman and A. Forsby, ATLA, Altern. Lab. Anim., 2007, 35, 33-38.

15 A. Avdeef, S. Bendels, L. Di, B. Faller, M. Kansy, K. Sugano and Y. Yamauchi, J. Pharm. Sci., 2007, 96, 2893-2909.

16 P. Artursson, K. Palm and K. Luthman, Adv. Drug Delivery Rev., 2001, 46, 27-43.

17 J. D. Irvine, L. Takahashi, K. Lockhart, J. Cheong, J. W. Tolan, H. Selick and J. R. Grove, J. Pharm. Sci., 1999, 88, 28-33.

18 H. Bohets, P. Annaert, G. Mannens, K. Anciaux, P. Verboven, W. Meuldermans and K. Lavrijsen, Curr. Top. Med. Chem., 2001, 1, 367-383.

19 P. Artursson and J. Karlsson, Biochem. Biophys. Res. Commun., 1991, 175, 880-885.

20 D. Newby, A. A. Freitas and T. Ghafourian, Eur. J. Med. Chem., 2015, 90, 751-765.

21 M.-C. Grès, B. Julian, M. Bourrié, V. Meunier, C. Roques, M. Berger, X. Boulenc, Y. Berger and G. Fabre, Pharm. Res., 1998, 15, 726-733.

22 V. Pade and S. Stavchansky, J. Pharm. Sci., 1998, 87, 16041607.

23 C. A. Lipinski, F. Lombardo, B. W. Dominy and P. J. Feeney, Adv. Drug Delivery Rev., 1997, 23, 3-25.

24 E. Deconinck, H. Ates, N. Callebaut, E. Van Gyseghem and Y. Vander Heyden, J. Chromatogr. A, 2007, 1138, 190-202.

25 T. Ghafourian, A. A. Freitas and D. Newby, Int. J. Pharm., 2012, 436, 711-720.

26 Y. H. Zhao, J. Le, M. H. Abraham, A. Hersey, P. J. Eddershaw, C. N. Luscombe, D. Boutina, G. Beck, B. Sherborne and I. Cooper, J. Pharm. Sci., 2001, 90, 749-784.

27 S. Agatonovic-Kustrin, R. Beresford and A. P. M. Yusof, J. Pharm. Biomed. Anal., 2001, 25, 227-237.

28 M. J. Polley, F. R. Burden and D. A. Winkler, Aust. J. Chem., 2006, 58, 859-863.

29 A. Yan, Z. Wang and Z. Cai, Int. J. Mol. Sci., 2008, 9, 19611976.

30 N. Basant, S. Gupta and K. P. Singh, Comput. Biol. Chem., 2016, 61, 178-196.

31 R. Jones, P. C. Connolly, A. Klamt and M. Diedenhofen, J. Chem. Inf. Model., 2005, 45, 1337-1342.

32 G. Klopman, L. R. Stefan and R. D. Saiakhov, Eur. J. Pharm. Sci., 2002, 17, 253-263. 
33 A. Talevi, M. Goodarzi, E. V. Ortiz, P. R. Duchowicz, C. L. Bellera, G. Pesce, E. A. Castro and L. E. Bruno-Blanch, Eur. J. Med. Chem., 2011, 46, 218-228.

34 M. Wen, B.-C. Deng, D.-S. Cao, Y.-H. Yun, R.-H. Yang, H.-M. Lu and Y.-Z. Liang, Analyst, 2016, 141, 5586-5597.

35 T. Hou, J. Wang and Y. Li, J. Chem. Inf. Model., 2007, 47, 2408-2415.

36 J. Shen, F. Cheng, Y. Xu, W. Li and Y. Tang, J. Chem. Inf. Model., 2010, 50, 1034-1041.

37 C. Suenderhauf, F. Hammann, A. Maunz, C. Helma and J. R. Huwyler, Mol. Pharmaceutics, 2010, 8, 213-224.

38 E. Deretey, M. Feher and J. M. Schmidt, Quant. Struct.-Act. Relat., 2002, 21, 493-506.

39 M. V. Varma, K. Sateesh and R. Panchagnula, Mol. Pharmaceutics, 2005, 2, 12-21.

40 M. De Vrieze, P. Janssens, R. Szucs, J. Van der Eycken and F. Lynen, Anal. Bioanal. Chem., 2015, 407, 7453-7466.

41 E. L. Cunha, C. F. Santos, F. S. Braga, J. S. Costa, R. C. Silva, H. A. Favacho, L. I. Hage-Melim, J. C. Carvalho, C. H. da Silva and C. B. Santos, J. Comput. Theor. Nanosci., 2015, 12, 36823691.

42 OECD-QSAR-07-ENV-JM-MONO, 2007, 2.

43 S. Tian, Y. Li, J. Wang, J. Zhang and T. Hou, Mol. Pharmaceutics, 2011, 8, 841-851.

44 T. A. Halgren, J. Comput. Chem., 1996, 17, 490-519.

45 J. Dong, D.-S. Cao, H.-Y. Miao, S. Liu, B.-C. Deng, Y.-H. Yun, N.-N. Wang, A.-P. Lu, W.-B. Zeng and A. F. Chen, J. Cheminf., 2015, 7, 1.

46 C. A. Lipinski, F. Lombardo, B. W. Dominy and P. J. Feeney, Adv. Drug Delivery Rev., 2012, 64, 4-17.
47 D.-S. Cao, Q.-S. Xu, Q.-N. Hu and Y.-Z. Liang, Bioinformatics, 2013, btt105.

48 D. S. Cao, Y. Z. Liang, J. Yan, G. S. Tan, Q. S. Xu and S. Liu, J. Chem. Inf. Model., 2013, 53, 3086-3096.

49 D. S. Cao, Y. N. Yang, J. C. Zhao, J. Yan, S. Liu, Q. N. Hu, Q. S. Xu and Y. Z. Liang, J. Chemom., 2012, 26, 7-15.

50 L. Breiman, Machine Learning, 2001, 45, 5-32.

51 V. Svetnik, A. Liaw, C. Tong, J. C. Culberson, R. P. Sheridan and B. P. Feuston, J. Chem. Inf. Model., 2003, 43, 1947-1958.

52 L. Breiman, J. Friedman, C. J. Stone and R. A. Olshen, Classification and regression trees, CRC press, 1984.

53 A. Liaw and M. Wiener, Chem. Eng. News, 2002, 2, 18-22.

54 M. D. Wessel, P. C. Jurs, J. W. Tolan and S. M. Muskal, J. Chem. Inf. Model., 1998, 38, 726-735.

55 Y. H. Zhao, M. H. Abraham, J. Le, A. Hersey, C. N. Luscombe, G. Beck, B. Sherborne and I. Cooper, Pharm. Res., 2002, 19, 1446-1457.

56 J. Borel, in Ciclosporin, Karger Publishers, 1986, pp. 9-18.

57 S. Omura, Macrolide antibiotics: chemistry, biology, and practice, Academic press, 2002.

58 F. W. Asselbergs, G. F. Diercks, H. L. Hillege, A. J. van Boven, W. M. Janssen, A. A. Voors, D. de Zeeuw, P. E. de Jong, D. J. van Veldhuisen and W. H. van Gilst, Circulation, 2004, 110, 2809-2816.

59 G. W. B. And and M. A. Murcko, J. Med. Chem., 1996, 39, 2887-2893.

60 Y. J. Xu and M. Johnson, J. Chem. Inf. Model., 2002, 42, 912926.

61 J. P. Bai, A. Utis, G. Crippen, H.-D. He, V. Fischer, R. Tullman, H.-Q. Yin, C.-P. Hsu, L. Jiang and K.-K. Hwang, J. Chem. Inf. Model., 2004, 44, 2061-2069. 